\title{
High redshift X-ray galaxy clusters
}

\section{The $L_{\mathrm{X}}-T$ relationship revisited}

\author{
M. Branchesi ${ }^{1,2}$, I. M. Gioia ${ }^{2}$, C. Fanti ${ }^{2}$, and R. Fanti ${ }^{2}$ \\ 1 Dipartimento di Astronomia, Università di Bologna, via Ranzani 1, 40127 Bologna, Italy \\ e-mail: m.branchesi@ira.inaf.it \\ 2 Istituto di Radioastronomia, INAF, Via Gobetti 101, 40129 Bologna, Italy \\ e-mail: [gioia;rfanti;cfanti]@ira.inaf.it
}

Received 13 March 2007 / Accepted 17 June 2007

\begin{abstract}
Aims. In this paper we re-visit the observational relation between X-ray luminosity and temperature for high- $z$ galaxy clusters and compare it with the local $L_{\mathrm{X}}-T$ and with theoretical models.

Methods. To these ends we use a sample of 17 clusters extracted from the Chandra archive supplemented with additional clusters from the literature, either observed by Chandra or XMM-Newton, to form a final sample of 39 high redshift $(0.25<z<1.3)$ objects. Different statistical approaches are adopted to analyze the $L_{\mathrm{X}}-T$ relation.

Results. The slope of the $L_{X}-T$ relation of high redshift clusters is steeper than expected from the self-similar model predictions and steeper, even though still compatible within the errors, than the local $L_{X}-T$ slope. The distant cluster $L_{X}-T$ relation shows a significant evolution with respect to the local Universe: high- $z$ clusters are more luminous than the local ones by a factor $\approx 2$ at any given temperature. The evolution with redshift of the $L_{X}-T$ relation cannot be described by a single power law nor by the evolution predicted by the self-similar model.

Conclusions. We find a strong evolution, similar or stronger than the self-similar model, from $z=0$ to $z \leq 0.3$ followed by a much weaker, if any, evolution at higher redshifts. The weaker evolution is compatible with non-gravitational models of structure formation. According to us a statistically significant sample of nearby clusters $(z<0.25)$ should be observed with the current available X-ray telescopes to completely exclude observational effects due to different generation detectors and to understand this novel result.
\end{abstract}

Key words. galaxies: clusters: general - galaxies: high-redshift - cosmology: observations - galaxies: intergalactic medium $\mathrm{X}$-rays: galaxies: clusters

\section{Introduction}

Clusters of galaxies represent the largest scale of fully collapsed structures in the Universe and thus offer a unique insight into the formation of structures and into the parameters governing their evolution. As powerful X-ray emitters $\left(L_{\mathrm{X}}=10^{43}-10^{45} \mathrm{erg} \mathrm{s}^{-1}\right)$ galaxy clusters can be easily traced up to high redshift, hence they are a very important observational tool for cosmologists. One problem that must be examined is how to relate the observable quantities (e.g., cluster X-ray gas temperature or luminosity) to the quantity predicted by the cosmological studies (usually mass). A detailed and accurate knowledge of the relations between observable bulk features is necessary. For instance, the relation between the X-ray luminosity and temperature of the intra-cluster medium (ICM), that is analyzed in detail in this paper, provides a means to convert an easily observed X-ray luminosity function into a more cosmologically useful mass function (e.g., Evrard et al. 1996; Reiprich \& Böhringer 2002; Evrard et al. 2002; Allen 2003; Borgani 2006).

Under the assumption that the baryons follow the total matter distribution (dominated by dark matter) Kaiser (1986) constructed the simple self-similar model of cluster formation where the ICM is driven only by gravitational processes like shocks and adiabatic compression. As long as the baryons are distributed in the same way as the total mass, each X-ray observable scales like some power of the mass. The self-similar model, supported by
$\mathrm{N}$-body hydrodynamical simulations which consider only gravitational processes (Bryan \& Norman 1998), predicts the relation between luminosity and temperature to be $L_{\mathrm{X}} \propto T^{2}$.

The observational tight correlation between luminosity and temperature found for low redshift cluster samples (e.g. Markevitch 1998; Arnaud \& Evrard 1999) indicates a similar formation history for all clusters and is qualitatively similar to the predictions of the self-similar models of the cluster formation. However the observed slope of the $L_{X}-T$ relation is found to be much steeper $(\simeq 3)$ than expected from the self-similar model. Such deviation from self-similarity is taken as evidence that the simple gravitational collapse is not the only process that governs the heating of baryons, and that non-gravitational processes occurring before or during the cluster formation contribute to the clusters energy budget in a non negligible way. To explain the departure from self-similarity an increase in the central entropy is required (e.g. Evrard \& Henry 1991; Kaiser 1991; Ponman et al. 1999; Tozzi \& Norman 2001). This extra entropy makes the gas harder to compress in the cluster core, particularly in the shallower potential wells of low temperature clusters, whose thermal bremsstrahlung emission is reduced as compared to the self-similar model prediction. The physical processes that can rise the entropy of the gas involve heating by supernovae and by active galactic nuclei (AGNs) (e.g. Balogh et al. 1999; Bower et al. 2001; Brighenti \& Mathews 2001; Borgani et al. 2002), or the removal of low-entropy gas via cooling (e.g. Bryan 2000; 
Voit \& Bryan 2001; Wu \& Xue 2002) or a combination of heating and radiative cooling (e.g. Voit et al. 2002; Tornatore et al. 2003; Borgani et al. 2004; Muanwong et al. 2006). Therefore an accurate analysis of the $L_{\mathrm{X}}-T$ relationship is also important to investigate the physics behind galaxy cluster formation.

Further information on the formation and evolution of structure in the Universe are given by the study of the statistical properties of high redshift cluster samples with respect to the low redshift samples. Any observed change in the $L_{X}-T$ scaling law at high redshift with respect to low redshift allows in principle to verify the predictions of the cluster self-similar model or to constrain different scenarios of heating schemes and cooling efficiency (e.g. Voit 2005a).

With the main goal to revisit the $L_{X}-T$ relationship for high- $z$ clusters we used seventeen clusters in the range $0.25<z<1.01$ selected from the Chandra archive. We analyzed the data as described in details in a companion paper (Branchesi et al. 2007a, Paper I). In order to increase the statistics the sample was supplemented with additional clusters from the literature with redshift up to $z \sim 1.3$, either observed by Chandra or XMM-Newton. The final sample consists of 39 objects. The observational results from the $L_{\mathrm{X}}-T$ analysis of the 39 distant clusters are compared with those obtained from the analysis of the $L_{X}-T$ relation of low- $z$ clusters (Markevitch 1998; Arnaud \& Evrard 1999) and with theoretical models for structure formation.

All the uncertainties in the paper are at the $1 \sigma$ confidence level, unless otherwise noted. We use a $\Lambda \mathrm{CDM}$ cosmology with $H_{0}=70 \mathrm{~km} \mathrm{~s}^{-1} \mathrm{Mpc}^{-1}$ and $\Omega_{\mathrm{m}}=1-\Omega_{\Lambda}=0.3$.

\section{Cluster scaling relations}

\subsection{The self-similar model}

The self-similar model detailed by Kaiser (1986) is the most natural expectation in a picture where clusters form via the collapse of the most dense regions and cluster baryons are heated only by gravitational processes (adiabatic compression and shock heating) during the collapse. In this scenario it is assumed that a constant fraction of the baryonic content of the Universe ends up in the intracluster gas in hydrostatic equilibrium within the dominant dark matter, and emits by thermal bremsstrahlung. A state of virial equilibrium is achieved within a virial radius $R_{\mathrm{v}}(z)$ which includes a mean density which is a factor $\Delta_{\mathrm{V}}(z)$ above the critical density of the Universe, $\rho_{c, z}=3 H_{z}^{2} /(8 \pi G)$, at that redshift. A cosmology factor $E_{z}$ is used hereafter to indicate the evolution of the Hubble constant at redshift $z$ with respect to $z=0$ for a flat cosmology with matter density $\Omega_{\mathrm{m}}$ :

$E_{z}=H_{z} / H_{0}=\left[\Omega_{\mathrm{m}}(1+z)^{3}+1-\Omega_{\mathrm{m}}\right]^{1 / 2}$.

In a flat $\Lambda=0$ cosmology $E_{z}=(1+z)^{1.5}$.

The density contrast is given by:

$\Delta_{\mathrm{V}}(z)=18 \pi^{2}+82\left(\Omega_{z}-1\right)-39\left(\Omega_{z}-1\right)^{2}$

with $\Omega_{z}=\Omega_{\mathrm{m}}(1+z)^{3} / E_{z}^{2}$ as in Bryan \& Norman (1998). In a flat $\Lambda=0$ cosmology $\Delta_{\mathrm{V}}=18 \pi^{2}$ and it is independent of redshift.

In this simple scenario, called the self-similar scenario, the properties of clusters of different masses and at different redshifts are related to one-another according to simple scaling laws. The expected properties of clusters at high redshift are identical to those of low-redshift clusters, apart from scaling factors reflecting the increase of the mean density of the Universe with redshift. The self-similar scenario predicts how the ICM physical properties, like luminosity and temperature, are related (e.g. Kaiser 1986, 1991; Evrard \& Henry 1991).

Under the assumption that the ICM is spherically symmetric, for a $\beta$-model (Cavaliere \& Fusco-Femiano 1976) the total mass within a radius $R$ is given by the following equation (see e.g. Voit 2005a):

$M_{\mathrm{tot}}(<R)=\frac{3 \beta k_{\mathrm{B}} T(\mathrm{~K}) R_{\mathrm{c}}}{G \mu m_{\mathrm{p}}} \frac{\left(R / R_{\mathrm{c}}\right)^{3}}{1+\left(R / R_{\mathrm{c}}\right)^{2}}$

where the core radius $R_{\mathrm{c}}$ and $\beta$ parametrize the gas density profile, $\mu$ is the mean molecular weight ( $\mu=0.6$ for a primordial composition with a $76 \%$ fraction contributed by hydrogen) and $m_{\mathrm{p}}$ the proton mass. The mass considered in the self-similar model, expressed as a function of the radius $R_{\Delta_{z}}$ which encloses a mean density which is a factor $\Delta_{z}$ above the critical density of the Universe, is given by:

$M_{\mathrm{tot}}\left(<R_{\Delta_{z}}\right)=\frac{4 \pi}{3} \rho_{\mathrm{c}, z} \Delta_{z} R_{\Delta_{z}}^{3}$

and:

$\Delta_{z}=\Delta(0) \Delta_{\mathrm{V}}(z) / \Delta_{\mathrm{V}}(0)$

where $\Delta(0)$ is the density contrast at $z=0$. From Eqs. (3) and (4) the radius corresponding to $\Delta_{z}$ is calculated as follows:

$R_{\Delta_{z}}=\sqrt{\frac{6 \beta k_{\mathrm{B}} T(\mathrm{~K})}{\mu m_{\mathrm{p}}} \frac{1}{H_{z}^{2} \Delta_{z}}-R_{\mathrm{c}}^{2}}$

where $H_{z}$ is defined in Eq. (1). For $\Delta_{z}=\Delta_{\mathrm{v}}(z)$ one obtains the virial radius $R_{\mathrm{v}}(z)$. These radii are $\propto T^{0.5}$ and decrease with redshift in a way dependent on the assumed cosmological model.

At very high energies the ICM behaves as a fully ionized plasma and thus it emits by thermal bremsstrahlung in the X-ray band. The emissivity for this thermal process at frequency $v$ scales as:

$\epsilon_{v} \propto n_{\mathrm{e}} n_{\mathrm{i}} g(v, T) T^{-1 / 2} \mathrm{e}^{-h v / k_{\mathrm{B}} T}$.

Ignoring the temperature dependence of the Gaunt factor $g(v, T)$ (see, e.g., Spitzer 1978), and assuming a constant ratio of gas density to total mass density, the scaling behavior of a cluster $\mathrm{X}$-ray bolometric luminosity can be written as:

$L_{\mathrm{bol}} \propto M \rho_{\mathrm{gas}} T^{1 / 2}$.

Then one can obtain the following scaling relations between the observed bolometric luminosity $L_{\text {bol }}$ and temperature of the gas $T$ and the total gravitating mass:

$L_{\mathrm{bol}} \propto T^{2} E_{z} \Delta_{z}^{1 / 2} \quad$ and $\quad L_{\mathrm{bol}} \propto M_{\mathrm{tot}}^{4 / 3} E_{z}^{7 / 3} \Delta_{z}^{7 / 6}$.

The relation between the two X-ray observables, temperature and luminosity, is a useful statistical tool that enables the study of the physics of clusters and allows the verification of the selfsimilarity model expectations.

\subsection{Evolution with redshift of the $L_{\mathrm{bol}}-T$ relation}

When a high-redshift cluster sample is analyzed, it is important to define what is meant by evolution, since different definitions are found in the literature. The self-similar models predict changes with redshift of the scaling laws through the factors $E_{z}$ and $\Delta_{z}$. For instance, the $L_{\mathrm{bol}}-T$ relation (Eq. (9)) contains 
the cosmological term $E_{z} \Delta_{z}^{1 / 2}$. One definition of evolution (hereafter referred to as $E v_{1}$ ) is any change with redshift in the scaling laws not accounted for by the $E_{z}$ and $\Delta_{z}$ factors. A second definition of evolution (hereafter referred to as $E v_{2}$ ) accounts for any change with respect to the local observed relations, including $E_{z}$ and $\Delta_{z}$.

In the case of $E v_{1}$ the approach to follow is to correct the $\mathrm{X}$-ray luminosity for the $E_{z}$ and $\Delta_{z}$ factors and then to introduce a factor $(1+z)^{A_{z}}$ to account for any remaining effect with redshift. The $L_{\mathrm{bol}}-T$ relation then becomes: $E_{z}^{-1}\left(\Delta_{z} / \Delta_{z=0}\right)^{-1 / 2} L_{\mathrm{bol}} \propto$ $T^{\alpha}(1+z)^{A_{z}}$. With this notation in the self-similar scenario $A_{z}=0$ and no $E v_{1}$ is present.

For $E v_{2}$ generally a factor $(1+z)^{A}$ is used which includes also the $E_{z}$ and $\Delta_{z}$ factors. For the $L_{\text {bol }}-T$ relation one would write: $L_{\mathrm{bol}} \propto T^{\alpha}(1+z)^{A}$. In an Einstein-de Sitter Universe the cosmological factor $E_{z}$ (Eq. (1)) and the overdensity $\Delta_{z}$ (Eq. (5)) are equal to $(1+z)^{1.5}$ and 1 , respectively. The self-similar scaling relation would be: $L_{\mathrm{bol}}=C T^{\alpha}(1+z)^{1.5}$, and therefore $A=1.5$. We would talk of evolution according to the definition of $E v_{2}$, but no evolution according to the definition of $E v_{1}$.

In a $\Lambda$ CDM model with $\Omega_{\mathrm{m}}=0.3$, the dependence of $E_{z} \Delta_{z}^{1 / 2}$ is no longer a power law of $(1+z)$ but it can be approximated with that law in a limited range of redshifts by the redshift dependent exponent $A$ :

$A=\frac{\log \left[\left(\frac{E_{z}}{E_{z=0}}\right)\left(\frac{\Delta_{z}}{\Delta_{z=0}}\right)^{1 / 2}\right]}{\log (1+z)}$, where $E_{z=0}=1$.

In the case of evolution $E v_{1}$ according to the redshift interval one can calculate the range of predictions for $A$ on the basis of the self-similarity model and evaluate any possible evolution with respect to self-similarity. For example for a redshift range $0.3 \div$ 1.0 the self-similar model prediction for $A$ is in the range $1 \div 1.1$.

\subsection{Breaking the self-similar model}

One way to break the scaling laws that predict $L_{\mathrm{bol}} \propto T^{2}$ is to have non gravitational energy injected into the intracluster medium before or during the cluster formation. An abundant literature exists on this subject (for a review see e.g. Voit 2005a; Tozzi 2006, and reference therein). The candidates for the energy excess are currently feedback from star formation processes and feedback from nuclear activity in the cluster galaxies. Another physical process that could break the simple self-similar scaling is the removal of low-entropy gas via cooling (e.g. Bryan 2000; Voit \& Bryan 2001).

For example, Voit (2005a) gives the following $L_{\mathrm{bol}}-T$ relations (his Eqs. (81)-(83)) for three different models:

$L_{\mathrm{bol}} \propto T^{3.5}\left(E_{z}\right)^{-1}$

$L_{\text {bol }} \propto T^{2.5}\left(E_{z}\right)^{-1} t(z)^{-1}$

$L_{\text {bol }} \propto T^{3}\left(E_{z}\right)^{-3} t(z)^{-2}$

where $t(z)$ is the cosmic time at redshift $z$. The above relations were obtained by using a cluster radius that encloses a mean density which is a fixed factor $(\Delta=200)$ with respect to the critical density. This alternative choice of a fixed overdensity is often found in the literature (see e.g. Evrard et al. 2002). In this case the factor $\Delta_{z}$ is constant and the redshift dependence in the selfsimilar scaling relations (Eq. (9)) is only through the factor $E_{z}$.

The first model is a pure pre-heating case, in which the minimum entropy is assumed to be independent of both cluster mass and redshift. The other two are cooling models. These models give a slope of the $L_{\mathrm{bol}}-T$ relation significantly steeper than the self-similar model. They also predict different dependences on redshift (approximately $\propto(1+z)^{-0.8},(1+z)^{0.4}$, and almost constant in the redshift range $0-1)$. The relations differ from the self-similar ones in their dependence on $T$ and on $z$. Therefore, in principle, any observed change in the scaling laws allows to constrain different scenarios of heating schemes and cooling efficiency.

\section{The cluster sample}

In this work we use Chandra archival data of eighteen distant $(0.25<z<1.01)$ clusters. Details of the X-ray analysis are given in a companion paper (Branchesi et al. 2007a, Paper I). This same sample was also used by Branchesi et al. (2007b) to check for the presence of overdensity of point sources in the inner region of clusters of galaxies.

Since the $L_{\text {bol }}-T$ relationship at both low and high redshift exhibits a large intrinsic scatter, mostly due to the strong cooling flow clusters (Fabian et al. 1994), the presence of cooling cores has to be considered. To reduce the intrinsic scatter, which precludes the accurate determination of the exact shape and of any possible evolution of the $L_{\mathrm{bol}}-T$ relation, one can exclude from the sample the clusters with strong cooling cores (e.g. Arnaud \& Evrard 1999; Ettori et al. 2004; Maughan et al. 2006). Alternatively, since cooling flow clusters are similar to non-cooling flow clusters except for the inner small cooling flow region, an approach to follow is to excise the cluster central cores and replace them with an extrapolation of the isothermal $\beta$-model before deriving cluster parameters (e.g. Markevitch 1998; Vikhlinin et al. 2002). There are four clusters in our sample which Vikhlinin et al. (2002) indicate as "possible cooling cor" clusters. We have kept these clusters in the sample without applying any correction to $T$ or to $L_{\text {bol }}$ since according to their radial profile derived by us these cooling cores, if present at all, are rather mild. A fifth cluster, ZW CL $1454.8+2233$ presents a very thermally complex ICM (see notes on individual clusters in Branchesi et al. 2007a, Paper I) and was thus excluded from the analysis. From now on the sample of 17 clusters is referred to as "17 cluster sample".

In order to increase the statistics we supplemented our data with the sample of Vikhlinin et al. (2002) (from now on VI02), Ettori et al. (2004) (from now on ET04) and of Maughan et al. (2006) (form now on MA06) after appropriate corrections. For clusters in common we use our own measurements. For clusters not in our sample but in common with the other three samples, we preferred the data of MA06 when available, since they use the most recent calibrations of Chandra. We also used some of the MA06 clusters which were observed with XMM-Newton. For clusters common to VI02 and ET04 samples, we use the VI02 clusters since their data are in better agreement with ours after corrections for the different radii are made (see Branchesi et al. 2007 a, Paper I). This choice was made "a posteriori" since the $\chi^{2}$ analysis evaluation of the combined data, given in Sect. 4, shows a worse value when using ET04 data, even if the final results do not change independently of the sample used. The final working sample is thus composed by 17 clusters from our sample, 8 clusters from VI02, 10 clusters from MA06 and 4 clusters from ET04 for a total of 39 clusters. From now on the sample of 39 clusters is referred to as "combined sample".

Since we want to compare our observational results with theoretical models, the choice of the radius within which integrated cluster properties are measured becomes very important, notably when a large range of redshits is involved. Several authors 
have chosen a constant radius which has the great advantage of simplicity. A constant radius would allow the analysis of the same fractional volume of clusters if clusters have all the same physical radius. However, in the self-similar models the virial radius is a function of redshift and temperature, thus a constant physical radius does not include a constant fraction of the cluster, and introduces a bias with redshift in the computed cluster luminosities. Since the self-similar models predict the outer boundary of the virialized part of clusters in terms of a density contrast $\Delta_{\mathrm{V}}(z)$ (see for example Bryan \& Norman 1998), we consider appropriate to calculate the sample luminosities using the radius where the mean enclosed density is a factor $\Delta_{z}$ above the critical density of the Universe (Ettori et al. 2004; Maughan et al. 2006). In this way a fixed fraction of virial radius is used, independent of the cluster redshift, with no redshift and temperature biases in the luminosities. This choice allows us to use in a straightforward way the dependence of cluster properties on redshift as reviewed earlier (see Sects. 2.1, 2.3). In the present analysis we use for the overdensity $\Delta_{z}=500 \times \Delta_{\mathrm{V}}(z) /\left(18 \pi^{2}\right)$ as in ET04. Note that the $\Delta_{z=0}$ value is $\approx 280$, while the overdensity in a flat $\Lambda=0$ cosmology would be 500. For each cluster the value of $R_{500}$ is calculated according to Eq. (6). All luminosities are extrapolated to $R_{500}$ using an isothermal $\beta$-profile.

The uncertainties on $R_{500}$ and $L_{500}$ are calculated using the propagation of the measurement uncertainties on temperature and luminosities (which are the dominant contribution) and of the uncertainties related to the $\beta$-model. Since core radius, $R_{\mathrm{c}}$, and $\beta$ are correlated quantities (surface-brightness model with large values of $R_{\mathrm{c}}$ and $\beta$ are similar to those with small values), the uncertainties on them are not independent. Thus the errors on $R_{\mathrm{c}}$ were ignored in the calculation of the uncertainties (see MA06).

It has to be noted that there may be some inconsistencies when comparing the high- $z$ combined sample with the local samples due to a different choice of the radius used for the luminosity estimates. Markevitch (1998) adopted a radius of $1.4 \mathrm{Mpc}$, independent of temperature and $\beta$, which is however close to $R_{500}$ for average temperatures and $\beta$ values due to the low redshift of their clusters. An evaluation of the effects due to different choices of the radius resulted in negligible differences as compared to the errors. Arnaud \& Evrard (1999) do not specify any radius. We guess that they used a value encompassing all the visible X-ray emission. Even in this case we expect that systematic effects are negligible.

The temperature and the $L_{500}$ luminosities for all the clusters used to analyze the $L_{\mathrm{bol}}-T$ relation are listed in Table 1 . It is worth to note that no dependence on redshift is present for either the luminosity or temperature of the sample clusters. The columns in Table 1 contain the following information:

- Column 1: cluster name. Asterisks indicate "possible cooling core" clusters as classified by VI02. However, no correction was applied to the four objects in the "17 cluster sample" since we checked that the cooling core, if any, is negligible.

- Column 2: spectroscopic redshift.

- Column 3: cluster temperature in keV. For VI02, ET04 and MA06 the temperature is taken from their papers.

- Columns 4, 5: core radius in kpc and $\beta$. For VI02, ET04 and MA06 these parameters are taken from their papers.

- Column 6: radius in $\mathrm{kpc}$ which corresponds to a redshiftdependent contrast $\Delta_{z}=500 \times \Delta_{\mathrm{v}}(z) /\left(18 \pi^{2}\right)$. For each cluster the radius $R_{500}$ is computed according to Eq. (6).

- Column 7: luminosity within $R_{500}$ expressed in $10^{44} \mathrm{erg} \mathrm{s}^{-1}$. For the "17 cluster sample" the estimated luminosities (see
Branchesi et al. 2007a, Paper I) are extrapolated to $R_{500}$ using a $\beta$-model. In the same way the VI02 luminosities (estimated within the fixed radius of $1.4 h_{70}^{-1} \mathrm{Mpc}$ ) and the MA06 luminosities (estimated within the radius $R_{200}$ corresponding to an overdensity $\left.\Delta_{z}=200 \times \Delta_{\mathrm{V}}(z) / \Delta_{\mathrm{V}}(0)\right)$ were extrapolated to $R_{500}$.

- Column 8: original sample.

\section{The $L_{\text {bol }}-T$ relationship for high-z clusters: the analysis method}

In order to examine how cluster luminosity scales with temperature and whether the $L_{\mathrm{bol}}-T$ relation depends on redshift, we followed four different steps in our analysis.

We first assumed the following evolution law $\left(E v_{2}\right)$ :

$L_{\mathrm{bol}, 44}=C T_{6}^{\alpha}(1+z)^{A}$

where $L_{\mathrm{bol}, 44}$ is the bolometric luminosity in units of $10^{44} \mathrm{erg} \mathrm{s}^{-1}$ and $T_{6}=T(\mathrm{keV}) / 6$.

The parameters $C, \alpha$ and $A$ are determined by a $\chi^{2}$ fit which takes into account the uncertainties on the luminosity and temperature $\left(\sigma_{L}, \sigma_{T}\right)$. The $\chi^{2}$ is written as:

$\chi^{2}=\sum_{i=1}^{N} \frac{\left[\log L_{\mathrm{bol}, 44, i}-\log C-\alpha \log T_{6, i}-A \log \left(1+z_{i}\right)\right]^{2}}{\epsilon_{L_{i}}^{2}+\alpha^{2} \epsilon_{T_{i}}^{2}}$

where $N$ is the number of clusters, $\epsilon_{T}$ is equal to $\sigma_{T_{6}} /\left(T_{6} \ln 10\right)$ and $\epsilon_{L}$ is equal to $\sigma_{L} /(L \ln 10)$. The denominator represents the propagation of the uncertainties on the measured quantities. The minimum $\chi^{2}$ is found by varying $\log C, A$ and $\alpha$ in a grid of values.

a) The fit is performed setting $A$ equal to zero. In this way we analyze the $L_{\mathrm{bol}}-T$ relation independently of the cluster redshift.

b) The three parameters $A, \alpha$ and $\log C$ are used as free parameters in the fit.

c) In order to constrain the evolution in the $L_{\mathrm{bol}}-T$ relation with respect to nearby galaxy clusters, an approach similar to VI02 and ET04 is adopted. The fit is performed by fixing in Eq. (12) $\alpha$ and $\log C$ to the best fit values $(\bar{\alpha}$, $\log \bar{C}$ ) obtained from two local samples, namely those by Markevitch (1998) and Arnaud \& Evrard (1999). Markevitch (1998) analyzes 30 nearby clusters with measured ASCA temperatures and ROSAT luminosities. His cluster luminosities are computed within a constant radius (1.4 Mpc for $H_{0}=70 \mathrm{~km} \mathrm{~s}^{-1} \mathrm{Mpc}^{-1}$ ). Arnaud \& Evrard (1999) analyze 24 nearby clusters with X-ray measurements from GINGA, ASCA and Einstein. Both samples have a median redshift of 0.05 . In our cosmology the $L_{\mathrm{bol}}-T$ slope and normalization found by Markevitch (1998) and Arnaud \& Evrard (1999) convert respectively to:

$\bar{\alpha}=2.64 \pm 0.27$ and $\log \bar{C}=0.80 \pm 0.04$

$\bar{\alpha}=2.88 \pm 0.15$ and $\log \bar{C}=0.77 \pm 0.03$.

As a last step the luminosities are scaled by the cosmological factor $E_{z}^{-1}\left(\Delta_{z} / \Delta_{z=0}\right)^{-1 / 2}$ as predicted by the self-similar model (see e.g. ET04 and MA06). The data are fitted using the following evolution law $\left(E v_{1}\right)$ :

$E_{z}^{-1}\left(\Delta_{z} / \Delta_{z=0}\right)^{-1 / 2} L_{\mathrm{bol}, 44}=C T_{6}^{\alpha} \cdot(1+z)^{A_{z}}$. 
Table 1. Combined sample: cluster parameters.

\begin{tabular}{|c|c|c|c|c|c|c|c|}
\hline Cluster name & $z$ & $T$ & $\bar{R} R_{\mathrm{c}}$ & $\bar{\beta}$ & $R_{500}$ & $L_{500}$ & Sample \\
\hline Abell 2125 & 0.246 & $3.4_{-0.2}^{+0.2}$ & $182 \pm 12$ & $0.54 \pm 0.02$ & $888 \pm 28$ & $2.13 \pm 0.08$ & "17 cluster" \\
\hline MS 1008.1-1224 & 0.302 & $6.0_{-0.3}^{+0.4}$ & $165 \pm 9$ & $0.64 \pm 0.02$ & $1241 \pm 42$ & $11.89 \pm 0.37$ & \\
\hline ZW CL $0024.0+1652^{*}$ & 0.394 & $4.4_{-0.4}^{+0.5}$ & $128 \pm 10$ & $0.67 \pm 0.03$ & $1003 \pm 58$ & $4.38 \pm 0.25$ & \\
\hline MS 1621.5+2640 & 0.426 & $7.5_{-0.7}^{+1.4}$ & $227 \pm 17$ & $0.65 \pm 0.03$ & $1255 \pm 84$ & $12.98 \pm 0.69$ & \\
\hline RXJ 1701.3+6414* & 0.453 & $4.5_{-0.3}^{+0.4}$ & $15 \pm 2$ & $0.41 \pm 0.01$ & $765 \pm 33$ & $7.14 \pm 0.40$ & \\
\hline CL $1641+4001$ & 0.464 & $5.1_{-0.7}^{+0.8}$ & $151 \pm 18$ & $0.77 \pm 0.06$ & $1092 \pm 91$ & $3.02 \pm 0.25$ & \\
\hline V $1524.6+0957$ & 0.516 & $5.0_{-0.5}^{+0.6}$ & $302 \pm 27$ & $0.80 \pm 0.05$ & $1029 \pm 72$ & $7.10 \pm 0.52$ & \\
\hline MS 0451.6-0305 & 0.539 & $9.4_{-0.5}^{+0.7}$ & $270 \pm 8$ & $0.90 \pm 0.02$ & $1505 \pm 51$ & $52.89 \pm 2.75$ & \\
\hline V $1121+2327$ & 0.562 & $4.5_{-0.4}^{+0.5}$ & $437 \pm 58$ & $1.19 \pm 0.18$ & $1108 \pm 116$ & $5.73 \pm 0.43$ & \\
\hline MS 2053.7-0449* & 0.583 & $4.3_{-0.4}^{+0.5}$ & $115 \pm 12$ & $0.64 \pm 0.03$ & $839 \pm 49$ & $5.81 \pm 0.55$ & \\
\hline V $1221+4918$ & 0.700 & $7.0_{-0.7}^{+0.8}$ & $272 \pm 20$ & $0.76 \pm 0.04$ & $1037 \pm 64$ & $13.22 \pm 0.79$ & \\
\hline MS 1137.5+6625 & 0.782 & $6.2_{-0.4}^{+0.5}$ & $116 \pm 6$ & $0.71 \pm 0.02$ & $910 \pm 39$ & $13.95 \pm 0.53$ & \\
\hline RDCSJ $1317+2911^{*}$ & 0.805 & $3.7_{-0.8}^{+1.4}$ & $61 \pm 16$ & $0.52 \pm 0.04$ & $592 \pm 87$ & $1.35 \pm 0.40$ & \\
\hline RDCSJ 1350+6007 & 0.805 & $4.1_{-0.6}^{+0.8}$ & $261 \pm 43$ & $0.70 \pm 0.07$ & $688 \pm 77$ & $5.33 \pm 0.78$ & \\
\hline RXJ 1716.4+6708 & 0.813 & $6.5_{-0.8}^{+0.9}$ & $119 \pm 11$ & $0.66 \pm 0.03$ & $882 \pm 61$ & $13.35 \pm 1.08$ & \\
\hline MS 1054.4-0321 & 0.830 & $8.3_{-0.7}^{+0.6}$ & $520 \pm 32$ & $1.38 \pm 0.11$ & $1339 \pm 89$ & $35.73 \pm 1.79$ & \\
\hline WARPJ $1415.1+3612$ & 1.013 & $6.2_{-0.7}^{+0.8}$ & $68 \pm 7$ & $0.60 \pm 0.02$ & $722 \pm 43$ & $12.27 \pm 0.88$ & \\
\hline CL 1416+4446* & 0.400 & $3.7_{-0.3}^{+0.3}$ & 152 & $0.66 \pm 0.04$ & $906 \pm 47$ & $4.14 \pm 0.29$ & VI02 \\
\hline MS $0302+1658^{*}$ & 0.424 & $3.6_{-0.5}^{+0.5}$ & 154 & $0.74 \pm 0.09$ & $929 \pm 88$ & $5.15 \pm 0.37$ & \\
\hline RASS $1347-114^{*}$ & 0.451 & $14.1_{-0.9}^{+0.9}$ & 84 & $0.65 \pm 0.01$ & $1706 \pm 56$ & $135.01 \pm 9.45$ & \\
\hline $3 \mathrm{C} 295^{*}$ & 0.460 & $5.3_{-0.5}^{+0.5}$ & 103 & $0.67 \pm 0.03$ & $1051 \pm 55$ & $8.04 \pm 0.56$ & \\
\hline MS 0016+1609 & 0.541 & $9.9_{-0.5}^{+0.5}$ & 274 & $0.74 \pm 0.02$ & $1396 \pm 41$ & $57.38 \pm 4.03$ & \\
\hline CL $0848+4456$ & 0.574 & $2.7_{-0.3}^{+0.3}$ & 78 & $0.58 \pm 0.04$ & $636 \pm 42$ & $1.29 \pm 0.09$ & \\
\hline RDCS $0910+5422$ & 1.100 & $3.5_{-0.7}^{+0.7}$ & 111 & $0.72 \pm 0.18$ & $550 \pm 92$ & $2.66 \pm 0.21$ & \\
\hline RDCS $0849+4452$ & 1.261 & $4.7_{-1.0}^{+1.0}$ & 119 & $0.85 \pm 0.33$ & $628 \pm 144$ & $2.95 \pm 0.22$ & \\
\hline RXJ 0542-4100 & 0.634 & $7.9_{-0.8}^{+1.0}$ & $132 \pm 17$ & $0.51 \pm 0.03$ & $982 \pm 63$ & $12.15 \pm 1.36$ & ET04 \\
\hline RXJ 2302+0844 & 0.734 & $6.6_{-0.6}^{+1.5}$ & $96 \pm 12$ & $0.55 \pm 0.03$ & $865 \pm 65$ & $5.45 \pm 0.17$ & \\
\hline RXJ 1252-2927 & 1.235 & $5.2_{-0.7}^{+0.7}$ & $77 \pm 13$ & $0.53 \pm 0.03$ & $532 \pm 40$ & $5.99 \pm 1.10$ & \\
\hline RXJ $0848+4453$ & 1.270 & $2.9_{-0.8}^{+0.8}$ & $163 \pm 70$ & $0.97 \pm 0.43$ & $499 \pm 115$ & $1.04 \pm 0.73$ & \\
\hline $\mathrm{ClJ} 0046.3+8530$ & 0.62 & $4.4_{-0.4}^{+0.5}$ & $137 \pm 28$ & $0.60 \pm 0.06$ & $792 \pm 56$ & $3.82 \pm 0.2$ & MA06 \\
\hline $\mathrm{ClJ} 1342.9+2828$ & 0.71 & $3.7_{-0.4}^{+0.5}$ & $172 \pm 26$ & $0.70 \pm 0.06$ & $724 \pm 55$ & $3.34 \pm 0.1$ & \\
\hline ClJ 1113.1-2615 & 0.73 & $4.7_{-0.7}^{+0.9}$ & $106 \pm 12$ & $0.67 \pm 0.04$ & $801 \pm 74$ & $3.73 \pm 0.3$ & \\
\hline ClJ 1103.6+3555 & 0.78 & $6.0_{-0.7}^{+0.9}$ & $141 \pm 18$ & $0.58 \pm 0.11$ & $807 \pm 93$ & $4.62 \pm 0.3$ & \\
\hline ClJ $0152.7-1357 \mathrm{~N}$ & 0.83 & $5.6_{-0.8}^{+1.0}$ & $249 \pm 48$ & $0.73 \pm 0.10$ & $820 \pm 93$ & $10.68 \pm 1.4$ & \\
\hline ClJ 0152.7-1357S & 0.83 & $4.8_{-1.0}^{+1.1}$ & $123 \pm 24$ & $0.66 \pm 0.07$ & $744 \pm 93$ & $6.42 \pm 0.9$ & \\
\hline ClJ $1559.1+6353$ & 0.85 & $4.1_{-1.0}^{+1.4}$ & $67 \pm 30$ & $0.59 \pm 0.09$ & $664 \pm 071$ & $2.47 \pm 0.3$ & \\
\hline $\mathrm{ClJ} 1008.7+5342$ & 0.87 & $3.6_{-0.6}^{+0.8}$ & $170 \pm 43$ & $0.68 \pm 0.09$ & $622 \pm 79$ & $3.57 \pm 0.3$ & \\
\hline $\mathrm{ClJ} 1226.9+3332$ & 0.89 & $10.6_{-1.1}^{+1.1}$ & $113 \pm 8$ & $0.66 \pm 0.02$ & $1070 \pm 58$ & $42.51 \pm 1.0$ & \\
\hline $\mathrm{ClJ} 1429.0+4241$ & 0.92 & $6.2_{-1.0}^{+1.5}$ & $97 \pm 9$ & $0.67 \pm 0.06$ & $806 \pm 90$ & $9.32 \pm 0.8$ & \\
\hline
\end{tabular}

d) The minimum $\chi^{2}$ is found either for $A_{z}$ equal to zero and leaving $A_{z}, \alpha$ and $\log C$ as free parameters.

With this approach the redshift dependence expected by the selfsimilar scenario is removed "a priori" and thus normalization and slope values consistent with those from the local relations should be obtained if the self-similar predictions on the evolution are correct.

\section{The $L_{\text {bol }}-T$ relationship for high-z clusters: results}

\subsection{Comparison with local samples}

The top panel of Fig. 1 shows the $L_{\mathrm{bol}}-T$ relationship for the "17 cluster sample" while the bottom panel shows the same plot for the "combined sample" of 39 clusters. Overplotted are the best-fit relationships found for the nearby clusters by Markevitch (1998) (dotted line) and Arnaud \& Evrard (1999) (dashed line). A displacement of the distant $L_{\mathrm{bol}}-T$ relation from the local ones is clearly evident, in agreement with the results of VI02, ET04 and MA06. High redshift clusters are on average systematically more luminous than the local ones by a factor $\approx 2$.

To quantify the differences between the high and low- $z$ clusters the $L_{\text {bol }}-T$ fitting procedure, described in steps a) and b), has been applied to the "17 cluster sample" and to the "combined sample". The results of all the fits are listed in Table 2. Setting $A=0$ (first and third line in Table 2), the best-fit values for the slope $\alpha$ are somewhat higher but still consistent within the errors with the local ones (see Eq. (13)) and significantly steeper than 

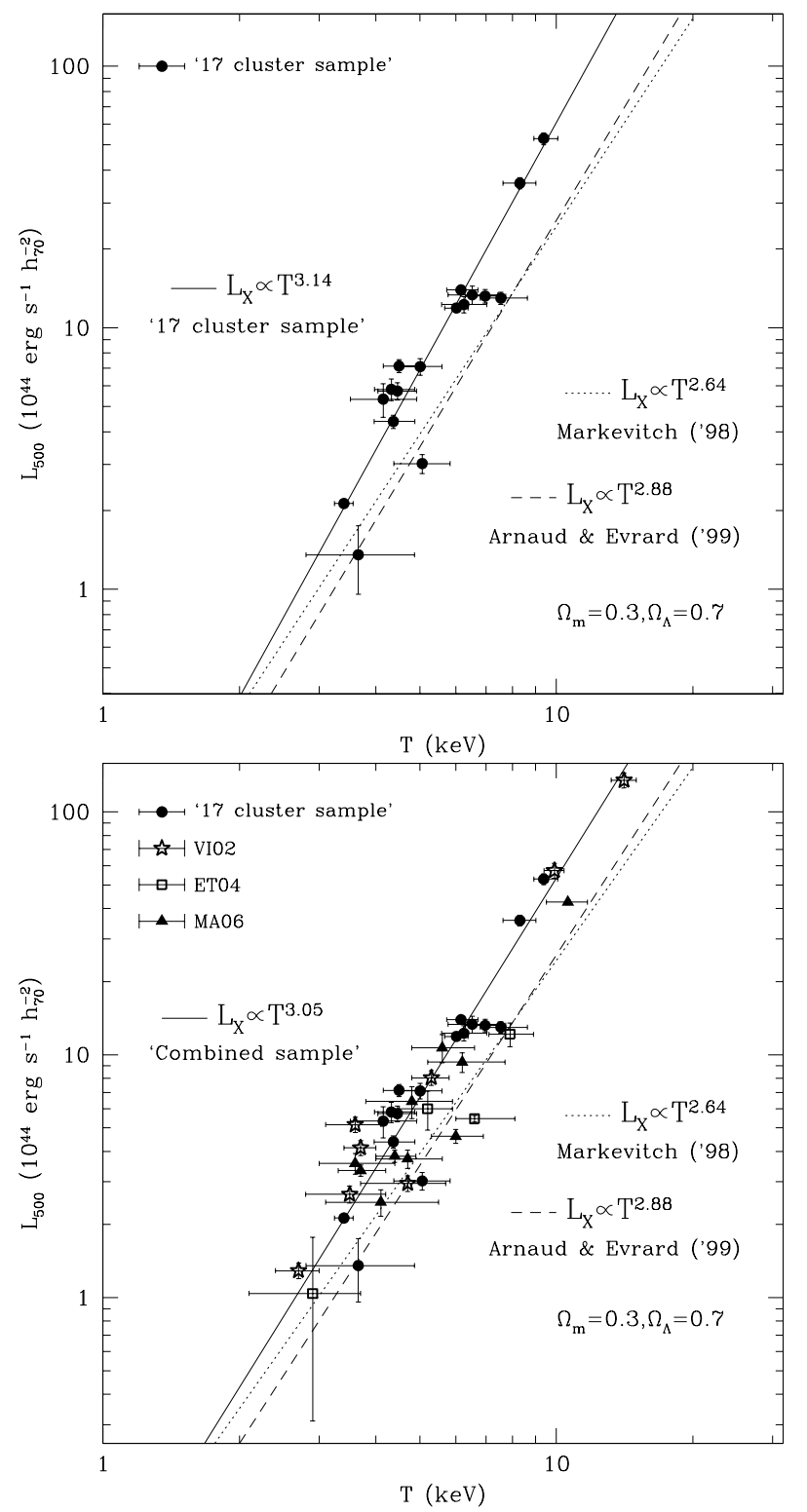

Fig. 1. (Top panel) The $L_{\mathrm{bol}}-T$ relation for the sample of 17 distant clusters. The solid line indicates the best fit to the data with $A$ set to 0 . Dotted and dashed lines refer to the nearby cluster samples. (Bottom panel) Same as above but for the "combined sample" of 39 clusters which includes data from MA06, VI02 and ET04 as explained in the text.

the self-similar predictions $(\alpha=2)$. The best-fit values for $\log C$ differ from the local ones at more than $5 \sigma$.

When the $E v_{2}$ evolution factor $(1+z)^{A}$ is introduced in the fit (second and fourth line in Table 2), the $\chi^{2}$ slightly improves but $\alpha$ and $\mathrm{C}$ do not change significantly suggesting that the dependence on redshift must be weak. In fact the $E v_{2}$ parameter $A$ does not differ significantly from zero. Therefore there is no evidence for a change in the normalization of the $L_{\mathrm{bol}}-T$ relation as a function of redshift in the range covered by the "combined sample" $(0.25 \leq z \leq 1.3)$ or, in other terms, no $E v_{2}$ is present.

The data were then fitted (step c) by fixing the slope and normalization to the best-fit relations obtained for the low- $z$ cluster samples of Markevitch (1998) and Arnaud \& Evrard (1999). The results are summarized in Table 3. A significant positive evolution (of $E v_{2}$ type) with respect to the local relations is required: $A$ varies between 1.2 and 1.35 for the "combined sample".
Table 2. Best fit results of $L_{\mathrm{bol}}-T$ relation - Luminosities extrapolated to $R_{500}$ (steps a and b)

\begin{tabular}{lcccc}
\hline \hline Sample & $A$ & $\alpha$ & $\log C$ & $\chi_{\min }^{2} /$ d.o.f. \\
\hline \multirow{2}{*}{17 cluster sample } & 0 & $3.14_{-0.29}^{+0.34}$ & $1.09_{-0.04}^{+0.04}$ & $12.18 / 15$ \\
& & & & \\
& $-0.08_{-1.22}^{+0.98}$ & $3.15_{-0.40}^{+0.57}$ & $1.10_{-0.18}^{+0.22}$ & $12.15 / 14$ \\
\hline \multirow{2}{*}{ combined sample } & 0 & $3.00_{-0.17}^{+0.19}$ & $1.06_{-0.03}^{+0.03}$ & $39.29 / 37$ \\
& & & & \\
& $-0.48_{-0.73}^{+0.58}$ & $3.03_{-0.20}^{+0.25}$ & $1.15_{-0.10}^{+0.15}$ & $37.18 / 36$ \\
\hline
\end{tabular}

Table 3. Best fit results of $L_{\mathrm{bol}}-T$ relation - Luminosities extrapolated to $R_{500}$ (step c)

\begin{tabular}{cccc}
\hline \hline Sample & $A$ & $\chi_{\min }^{2}$ /d.o.f. & Reference sample \\
\hline 17 cluster sample & $1.40_{-0.13}^{+0.13}$ & $22.39 / 16$ & Markevitch (1998) \\
& $1.58_{-0.15}^{+0.13}$ & $23.26 / 16$ & Arnaud \& Evrard (1999) \\
\hline & & & \\
combined sample & $1.20_{-0.08}^{+0.08}$ & $73.57 / 38$ & Markevitch (1998) \\
& $1.35_{-0.10}^{+0.08}$ & $68.35 / 38$ & Arnaud \& Evrard (1999) \\
\hline
\end{tabular}

The slope $(\alpha)$ and the normalization $(\log C)$ are fixed to the best-fit values obtained for the low- $z$ cluster sample indicated in the last column.

A similar positive evolution is found by other authors (e.g. VI02; Kotov \& Vikhlinin 2005; Lumb et al. 2004; and MA06) ${ }^{1}$. However, the best-fits are significantly worsened with respect to the previous step $b$ ) analysis. In particular the $\chi^{2}$ of the fit for the "combined sample" has a probability lower than $0.1 \%$ to be acceptable. This evolution, which would be consistent with the self-similar model predictions $(A \approx 0.9 \div 1.2$ for the redshift range $0.25 \div 1.3$ with respect to redshift 0 ), is not found in the high- $z$ sample, but only when the entire redshift range $(0 \leq z \leq 1.3)$ is considered, that is using the local samples as well.

In order to emphasize this result, we present in Fig. 2 the ratios between the observed cluster luminosity and the "expected luminosity" derived using the observed high- $z$ cluster temperature in the local relations by Markevitch (1998) and Arnaud \& Evrard (1999) as a function of $(1+z)$. For each cluster of the "combined sample" the "expected luminosity" is calculated as:

$L_{\text {Markevitch,Arnaud }}^{\text {expected }}=\bar{C} T_{6}^{\bar{\alpha}}$

where $\alpha$ and $C$ are fixed to the best-fit values obtained by Markevitch (1998) and Arnaud \& Evrard (1999) (see Eq. (13)), respectively, and $T$ is the measure of the high- $z$ cluster temperature. Clearly the evolution law fitted according to step c) (see Table 3) and indicated by the solid line is not a good representation of the data.

1 VI02 and Kotov \& Vikhlinin (2005) found $A=1.5$ and $A=1.8$, respectively, somewhat larger than our value. This is largely accounted for by their different choice for the radius $(1.4 \mathrm{Mpc})$ within which $L_{\mathrm{bol}}$ is computed. Using their same radius and comparing our sample with Markevitch (1998), we find $A=1.6$ in good agreement with them. 

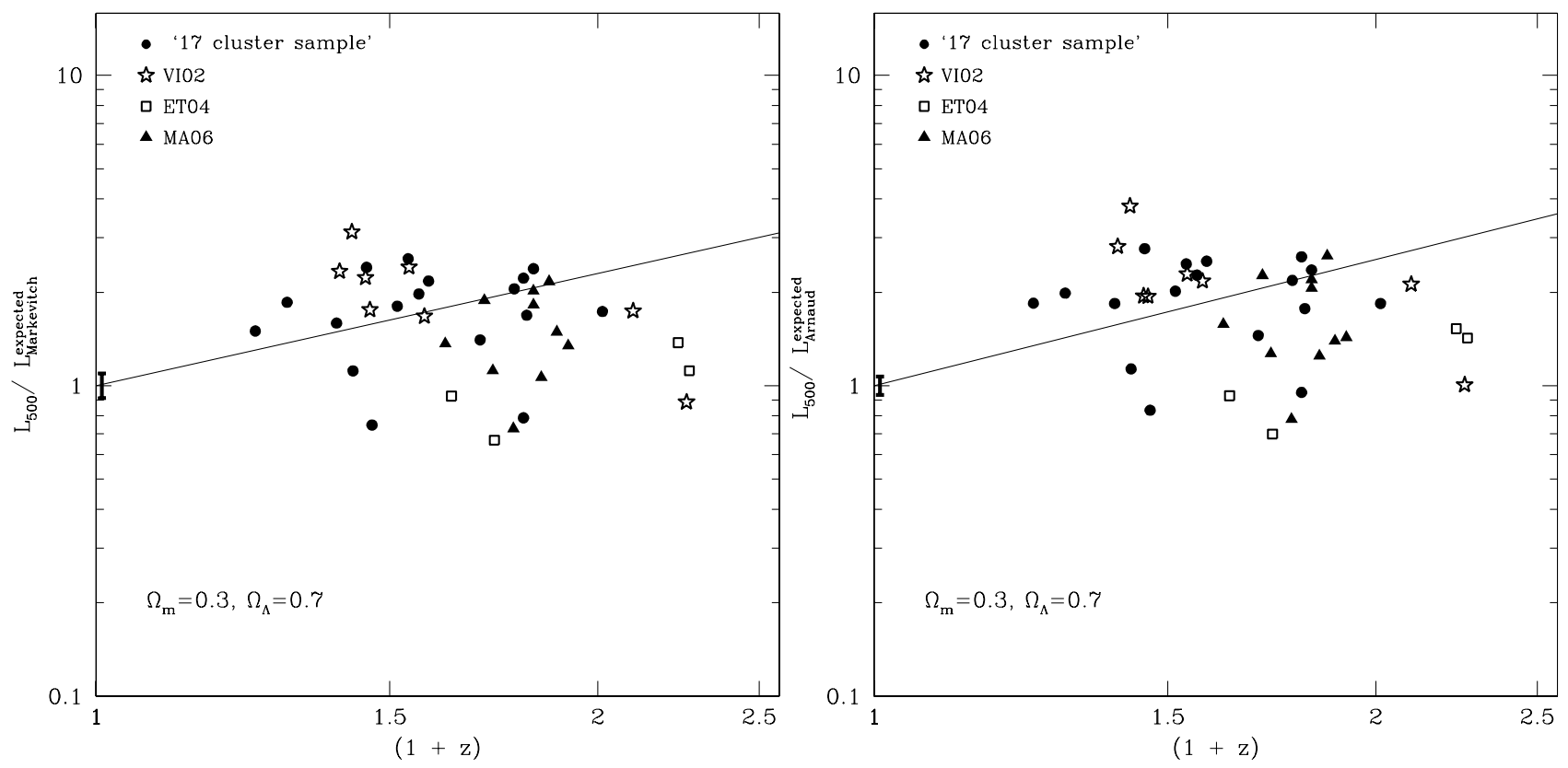

Fig. 2. Ratio of the observed luminosity to the "expected luminosity" from the local relations (see Sect. 5.1) versus $(1+z)$ for the "combined sample". (Left panel) The expected luminosity $\left(L_{\text {Markevitch }}^{\text {expete }}\right)$ is the luminosity obtained from the local relation of Markevitch (1998) using the observed cluster temperature. (Right panel) Same as above but for the local relation of Arnaud \& Evrard (1999). In both panels the solid line indicates the fitted evolution law according to step c) of the Sect. 4 . The evolution laws are clearly not appropriate. The error bar at $z=0$ indicates the uncertainty on the normalization of the local sample best-fit law.

Table 4. Best fit results of $E_{z}^{-1}\left(\Delta_{z} / \Delta_{z=0}\right)^{-1 / 2} L_{\mathrm{bol}}-T$ relation (step d).

\begin{tabular}{lcccc}
\hline \hline Sample & $A_{z}$ & $\alpha$ & $\log C$ & $\chi_{\min }^{2}$ /d.o.f. \\
\hline 17 cluster sample & 0 & $2.93_{-0.28}^{+0.33}$ & $0.89_{-0.04}^{+0.04}$ & $17.99 / 15$ \\
& & & & \\
& $-1.28_{-1.22}^{+0.98}$ & $3.17_{-0.43}^{+0.55}$ & $1.13_{-0.18}^{+0.23}$ & $12.18 / 14$ \\
\hline combined sample & 0 & $3.00_{-0.18}^{+0.19}$ & $0.87_{-0.03}^{+0.03}$ & $61.09 / 37$ \\
& & & & \\
& $-1.80_{-0.65}^{+0.70}$ & $3.05_{-0.23}^{+0.23}$ & $1.20_{-0.13}^{+0.13}$ & $37.37 / 36$ \\
\hline
\end{tabular}

The luminosities extrapolated to $R_{500}$ correspond to a redshiftdependent contrast of 500, and were scaled by the cosmological factor $E_{z}^{-1}\left(\Delta_{z} / \Delta_{z=0}\right)^{-1 / 2}$ as predicted by the self-similar model.

\subsection{Comparison with the self-similar model}

In Table 4 we list the results of the fits obtained by scaling the luminosities by the cosmological factor $E_{z}^{-1}\left(\Delta_{z} / \Delta_{z=0}\right)^{-1 / 2}$, as described in step d), and fixing $A_{z}=0$. The best fit values of parameters $\alpha$ and $C$ are found to be very similar to those of the low redshift clusters. This result is illustrated in Fig. 3 where the high redshift cluster data points, corrected for the cosmological factor, are randomly distributed on the low $-z$ relations (see also ET04 and MA06). However the $\chi^{2}$ value in Table 4 for the "combined sample" shows that the fit is not good (probability lower than $1 \%$ to be acceptable). This is emphasized in Fig. 4 that shows the ratios between the luminosity scaled by the cosmological factor $E_{z}^{-1}\left(\Delta_{z} / \Delta_{z=0}\right)^{-1 / 2}$ and the luminosity corresponding to the cluster temperature according to the local relations as a function of $(1+z)$ for the "combined sample". If the evolution were consistent with the predictions of the self-similar models, then the data points should be randomly distributed around unity, independent of the redshift. Figure 4 shows instead an excess of clusters above unity for $z \leq 0.6$ and a large excess below unity for $z>0.6$.

When $A_{z}$ is let free to vary we find a strong negative $E v_{1}$ evolution with the normalization factor $\mathrm{C}$ increasing by $\approx \mathrm{a}$ factor 2 (see Table 4) which brings the data points to shift back, away from the local relations. This is the same effect mentioned earlier. In order to bring the high- $z$ clusters data points in agreement with the local relation a redshift dependent correction must be applied to the data. This effect is not present in the redshift range explored by the combined sample of 39 clusters.

We are led to think that the dependence on redshift of the $L_{\text {bol }}-T$ relation in the full range $0<z<1.3$ cannot be described by either the factor $E_{z}\left(\Delta_{z} / \Delta_{z=0}\right)^{1 / 2}$ or by a power law factor $(1+z)^{A}$. These contradictions between data and evolution models $\left(E v_{1}\right.$ and $\left.E v_{2}\right)$ were already noted by Voit (2005b) who used a collection of published data. Voit pointed out that the luminosity evolution can not be monotonic with redshift. He suggested that the self-similar evolution may be plausible at low redshift, but cannot continue to arbitrarily high redshift. This might be consistent with radiative cooling and feedback from galaxy formation being increasingly more important at high redshift.

Unless there are systematic observational effects between the low- $z$ and high- $z$ cluster data (for instance a systematic temperature difference of $\approx 25 \%$ or an appropriate combination of systematic differences in luminosity and temperature) our results require a strong evolution, similar or stronger than the self-similar, from $z=0$ to $z \leq 0.3$ followed by a null evolution at higher redshift. Systematic differences in both luminosity and temperature between past and current generation of X-ray detectors are not reported in the literature. Vikhlinin et al. (2002) specifically verified that no bias is present between the temperatures measured by Chandra and by $A S C A$.

An additional effect could be the presence of the Malmquist bias in flux-limited samples (Sect. 5.2 in Maughan 2007). 

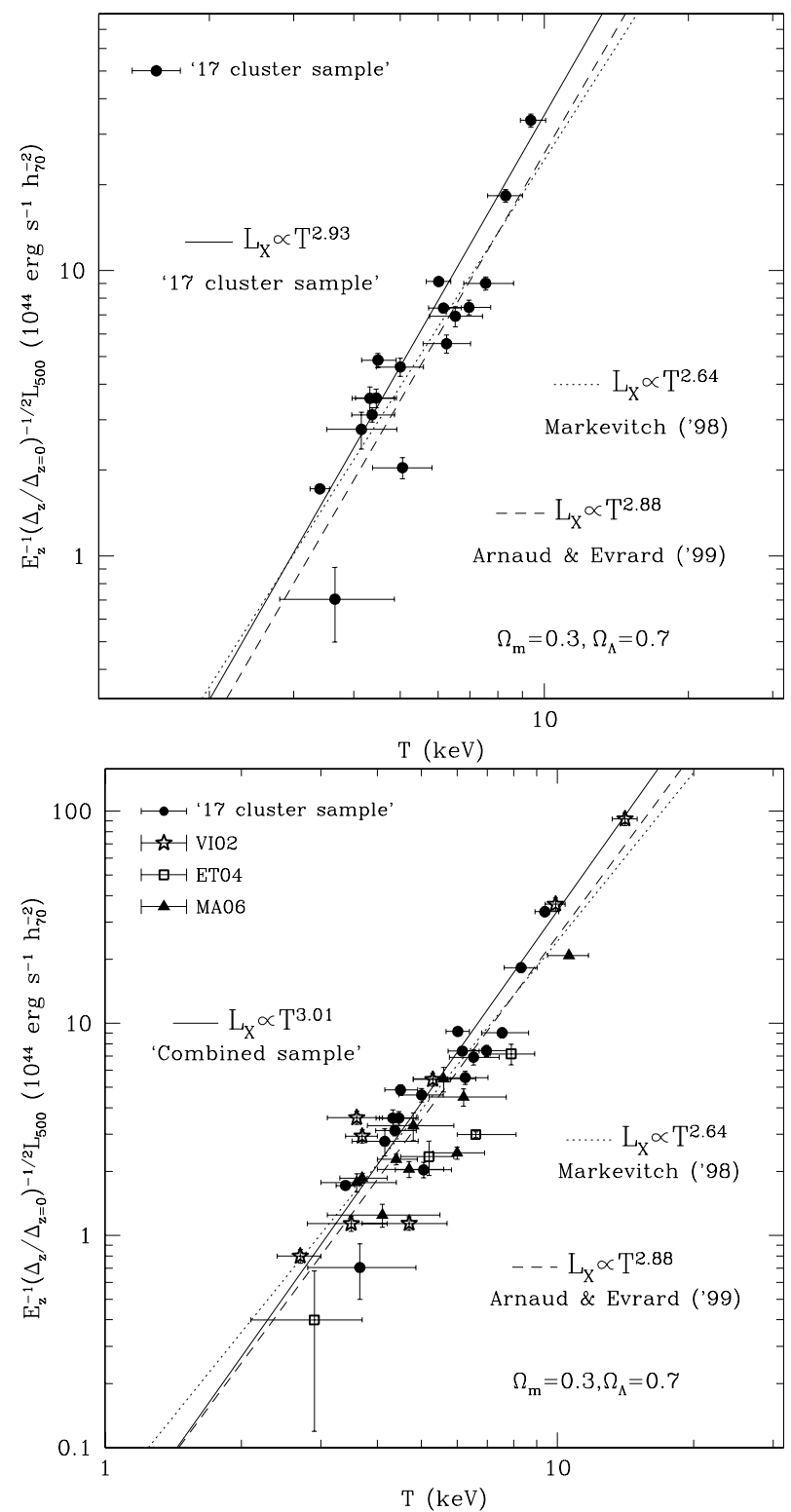

Fig. 3. The $E_{z}^{-1}\left(\Delta_{z} / \Delta_{z=0}\right)^{-1 / 2} L_{\mathrm{bol}}-T$ relation for the " 17 cluster sample" (top panel) and for the combined 39 cluster sample (bottom panel). The luminosity of each cluster is scaled by the cosmological factor $E_{z}^{-1}\left(\Delta_{z} / \Delta_{z=0}\right)^{-1 / 2}$. The solid line indicates the best-fit to the data obtained fixing $A_{z}=0$. The dotted and dashed lines indicate the local relations.

However, this effect is very difficult to quantify with the existing heterogeneous archival cluster samples.

\subsection{Comparison with additional Chandra data}

A very recent work by Maughan et al. (2007) analyzes a sample of 115 galaxy clusters at $0.1<z<1.3$ observed with Chandra. It is interesting for us to use the Maughan et al. (2007) sample since they have a large number of nearby objects $(z<0.4)$ which are missing in our sample. From their 115 clusters we selected 56 clusters as follows. Twenty-five objects are in common with our "combined sample" clusters and, according to us, are not contaminated by the central "cooling core". These 25 clusters were used to verify the presence of systematic difference between ours and Maughan measurements. Our estimates of the cluster temperature and luminosity are on average $15 \%$ and $10 \%$ higher with respect to Maughan et al. (2007). We do not discuss here the possible reasons for these systematic differences, but just take them into account in the analysis. The remaining clusters were selected from the $z<0.4$ sample and are those clusters for which the ratio between the luminosities with and without the central cluster region is less than 1.7 (see Cols. 4 and 8 of Table 3 in Maughan et al. 2007). In such a way we exclude clusters with strong cooling cores. Figure 5 is the same as Fig. 2 but for the Maughan's cluster measurements. A comparison between Figs. 5 and 2 shows that the two distributions are very similar ${ }^{2}$ and confirms and extends to lower redshift our results. In fact the $z>0.25$ clusters have on average a flat distribution higher than $L_{\text {Maughan }} / L_{\text {Markevitch }}^{\text {expected }}=1$ which is the local cluster value. At lower redshift $(z<0.25)$ the region pertaining to the local samples begins to being suddenly populated ( 8 clusters). The reason of this change in the distribution is not at all clear and not accounted for to our knowledge by any model.

\subsection{Comparison with non-gravitational models}

Figure 6 shows the comparison between the high- $z$ "combined sample" and the three non-gravitational models described by Voit (2005a) where the effects of preheathing and radiative cooling are included in the simple self-similar scenario (see Sect. 2.3). To properly compare our data to the Voit models the luminosities were extrapolated to a radius corresponding to a fixed overdensity $(\Delta=200)$ with respect to the critical density as required by the models. The three panels in Fig 6 give the ratio of the extrapolated luminosity to the $T^{\alpha}$, where $\alpha$ is the slope of the $L_{\text {bol }}-T$ in each model versus $(1+z)$. The dashed lines represent the fit of the models to the data, the dot-dashed lines represent the case when the models are normalized to the local samples at $z=0$.

It is worth noting that if only the "combined sample" $(0.25 \leq$ $z \leq 1.3$ ) is considered the three models (dashed lines in Fig. 6) are in agreement with the cluster data. The model that better describes the redshift evolution of the $L_{\mathrm{bol}}-T$ relation is the third model $\left(L_{\mathrm{bol}} \propto T^{3}\left(E_{z}\right)^{-3} t(z)^{-2}\right)$ shown in the bottom panel of Fig. 6. The corresponding minimum $\chi^{2}$ has a probability of about $50 \%$ to be acceptable $\left(\chi_{\min }^{2} /\right.$ d.o.f. $\left.=37.3 / 38\right)$ against a probability of about $5 \%$ for the other models (model 1 $\chi_{\min }^{2} /$ d.o.f. $=51.8 / 38, \operatorname{model} 2 \chi_{\min }^{2} /$ d.o.f. $\left.=57.3 / 38\right)$. The third model has also the same temperature slope $(\alpha=3)$ as the fit of a power law $\left(L_{\Delta=200}=C T_{6}^{\alpha}(1+z)^{A}\right)$ to the data (solid line in the bottom panel of Fig. 6).

When we link the high- $z$ "combined sample" to the local samples (see dot-dashed lines in Fig. 6) none of these model is acceptable. Namely none of the non-gravitational models seems to be able to account for the redshift dependence in the entire redshift range $0 \leq z \leq 1.3$.

\section{Summary and conclusions}

In this paper we have presented a re-visitation of the observational relation between X-ray luminosity and temperature for high- $z$ clusters of galaxies. To this purpose we have constructed the $L_{\text {bol }}-T$ relation for a sample of 17 distant clusters $(0.25<$ $z<1.01)$ selected from the Chandra archive. A detailed analysis of the X-ray data are given in a companion paper (Branchesi et al. 2007a, Paper I). The statistics was increased by adding

2 The systematic differences in luminosity and temperature mentioned above convert into a higher normalization (about 25\%) in the $y$-axis of the Maughan distribution with respect to ours. 

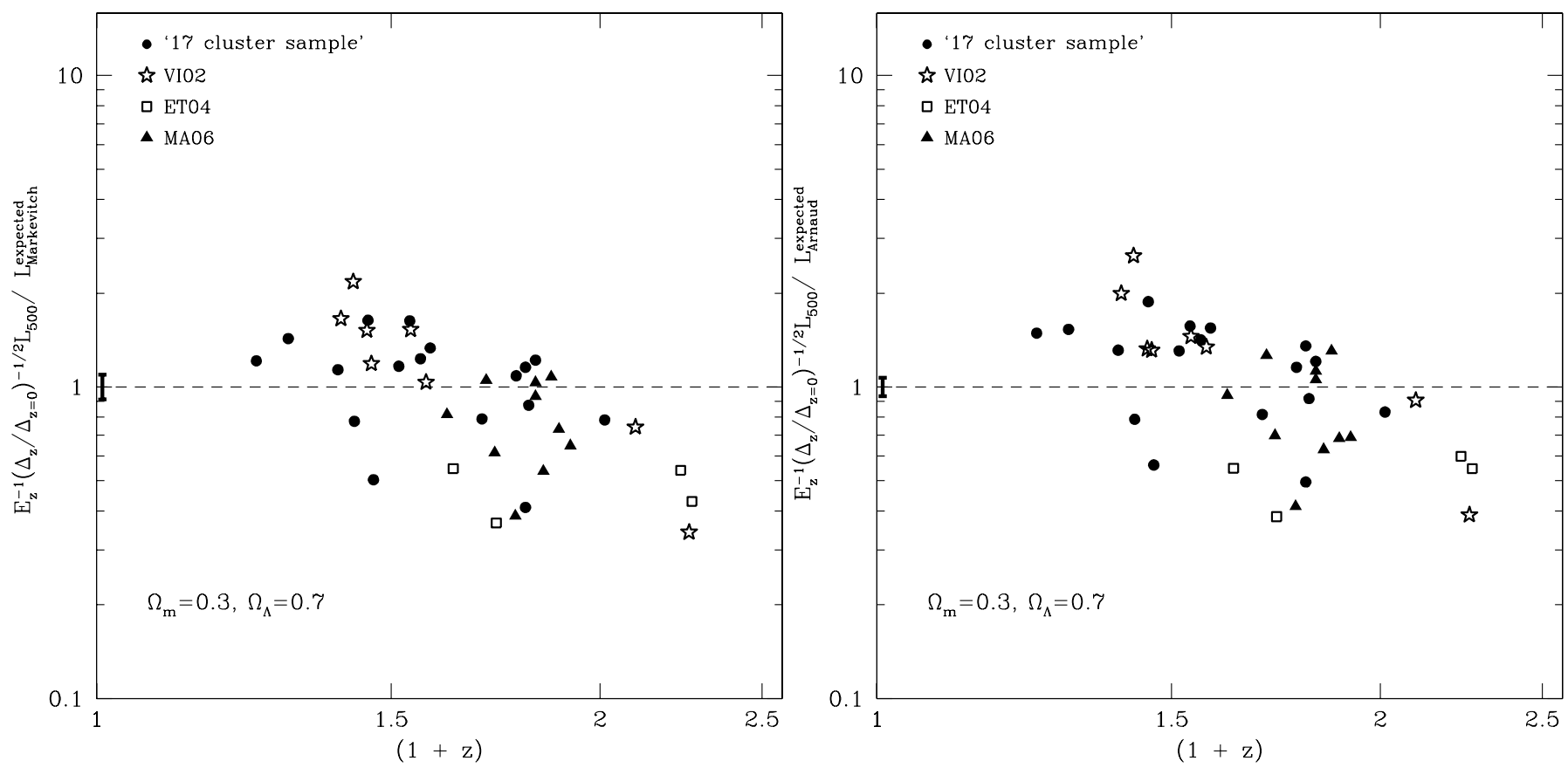

Fig. 4. (Left panel) Plot of the ratio between the luminosity scaled by the cosmological factor $E_{z}^{-1}\left(\Delta_{z} / \Delta_{z=0}\right)^{-1 / 2}$ and the "expected luminosity" derived using the cluster temperature in the local relation of Markevitch (1998) versus $(1+z)$ for the "combined sample". (Right panel) Same as above but for the local relation of Arnaud \& Evrard (1999). In both panels the error bar at $z=0$ indicates the uncertainty on the normalization of the local sample best-fit law.

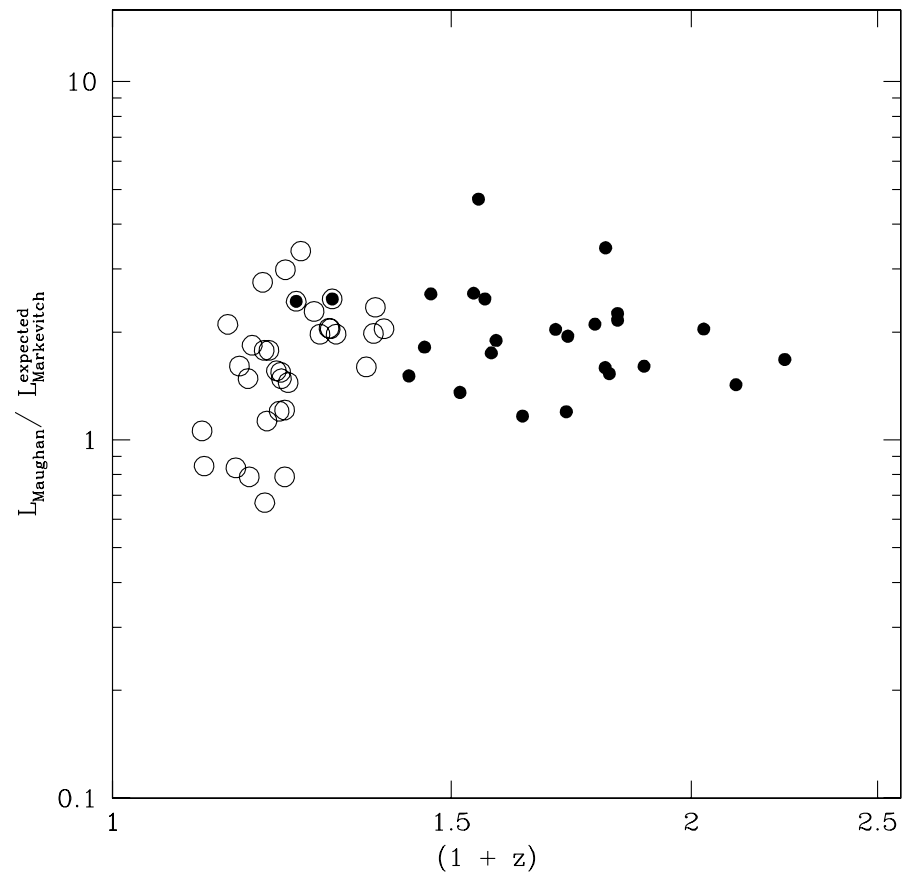

Fig. 5. Ratio of the luminosity measured by Maughan et al. (2007) to the "expected luminosity" derived using the Maughan et al. (2007) measured temperature in the local relations by Markevitch (1998) for the 56 clusters (see text for details). Filled circles indicate the 25 objects in common with our "combined sample". Open circles are objects with $z<0.4$ and with a ratio between the luminosities with and without the central cluster region less than 1.7 (see Cols. 4 and 8 of Table 3 in Maughan et al. 2007).

to our sample 22 clusters taken from the literature to form a final sample of 39 objects in the redshift range $0.25<z<1.3$, observed either by Chandra or XMM-Newton. The analysis of the larger sample allows a more stringent determination of the

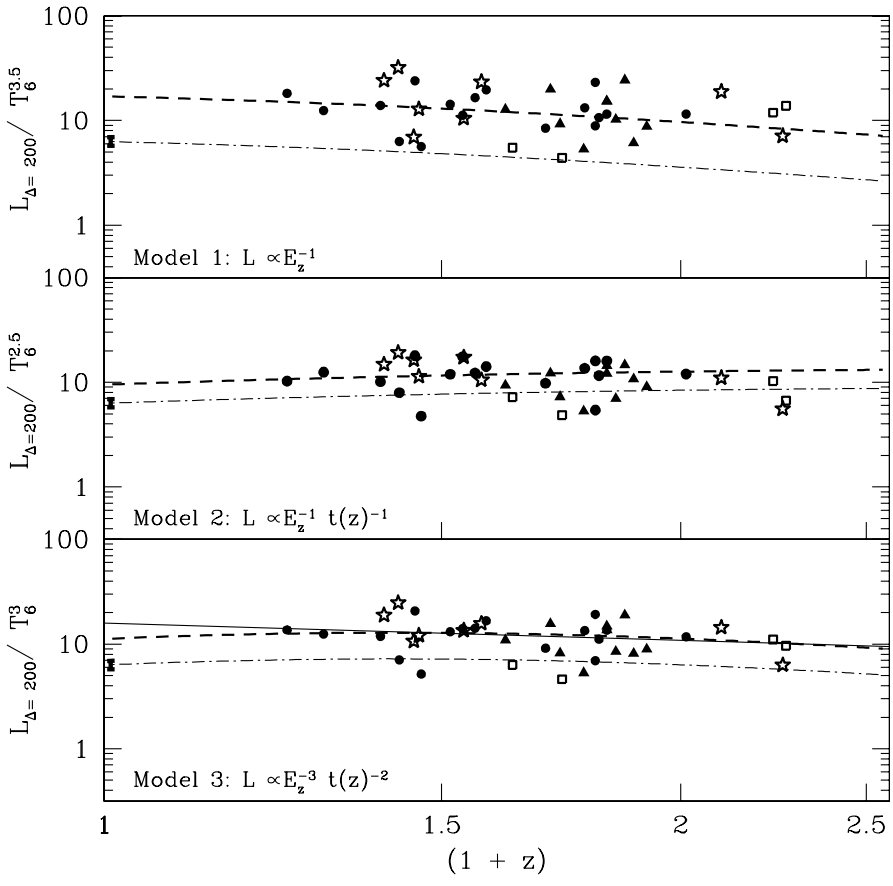

Fig. 6. Ratio between the $L_{\Delta=200}$ and $T^{\alpha}$, where $\alpha$ is the temperature dependence of the $L_{\mathrm{bol}}-T$ relation found by Voit (2005a), versus $(1+z)$. The dashed lines indicate the redshift dependence of the nongravitational models, considering only the "combined sample". The dot-dashed lines represents the non-gravitational models when they are normalized to the local samples. The solid line in the bottom panel represents the fit obtained following step b) described in Sect. 4 and using $L_{\Delta=200}(A=-0.55 \pm 0.66, \alpha=3.00 \pm 0.22, \log C=1.20 \pm 0.13$, $\chi_{\min }^{2} /$ d.o.f. $\left.=1.01\right)$. The error bar at $z=0$ in each panel indicates the normalization of the local samples.

high- $z L_{\mathrm{bol}}-T$ relation for a comparison with the local one and with the theoretical models. We emphasize the importance to 
extrapolate the luminosities to a fixed fraction of the virial radius when observational results are compared to theoretical predictions. The present analysis indicates that the apparently different results on the $L_{\mathrm{bol}}-T$ found by several authors can be in some cases explained by the different methods used, for instance a different choice of the cluster radius, a different definition of evolution $\left(E v_{1}\right.$ or $\left.E v_{2}\right)$ and/or a different way to compare the observational results with the self-similar predictions.

Our analysis of the $L_{\mathrm{bol}}-T$ relation of high redshift clusters has revealed a significant evolution with respect to the local Universe. The evolution goes in the direction of high- $z$ clusters being brighter than the local ones by a factor $\approx 2$ at any given temperature. The slope of the relationship is found to be steeper than expected from the self-similar model predictions and steeper, even though still compatible within the errors, than the local slope (Markevitch 1998; Arnaud \& Evrard 1999).

For the analysis of the $L_{\mathrm{bol}}-T$ evolution with redshift, different approaches were adopted (see Sect. 4):

- the data corresponding to the distant clusters in the redshift range $0.25 \leq z \leq 1.3$ were fitted considering the evolution factor $(1+z)^{A}$;

- a second fit is performed with the evolution factor $(1+z)^{A}$ and fixing the slope and normalization to the best-fit parameters obtained for the well studied low- $z$ cluster relations;

- the luminosities are corrected for the cosmological factor predicted by the self-similar model $E_{z}^{-1}\left(\Delta_{z} / \Delta_{z=0}\right)^{-1 / 2}$ before the fit is performed.

The following results are found:

i) The high- $z$ "combined sample" shows no significant changes with $z$ in the explored range $0.3 \leq z \leq 1.3$. The fit of the data with a power law $(1+z)^{A}$ yields for A a value not significantly different from zero.

ii) The self-similar model is not consistent with the data.

iii) When the high- $z$ "combined sample" is linked to the local samples no acceptable fit is obtained either using a power law $(1+z)^{A}$ or the self-similar scaling law.

The data from a recent paper by Maughan et al. (2007) confirm our results down to $z \simeq 0.25$. In addition, we note in the range $0.1<z<0.25$ the appearance of a number of clusters with the same properties as the local clusters.

The non-gravitational models by Voit (2005a) are in good agreement with the data when the redshift range $0.3<z<1.3$ is considered. When the entire range $0<z<1.3$ is taken into account none of these models is acceptable.

Summarizing we find that the dependence of the $L_{X}-T$ relation on redshift in the full redshift range explored $(0 \leq z \leq 1.3)$ cannot be described by the factor $E_{z}\left(\Delta_{z} / \Delta_{z=0}\right)^{1 / 2}$ of the selfsimilar model, or by any power law of the form $(1+z)^{A}$. It seems that a strong evolution is required by the $L_{X}-T$ relation from $z=0$ to $z \sim 0.3$ followed by a much weaker, if any, evolution at higher redshift. This weaker evolution is compatible with an increasing importance at high redshift of non-gravitational effects in the structure formation process (Voit 2005b).

According to us the first priority for a correct analysis of the evolution of the $L_{\mathrm{bol}}-T$ relation is to assemble a more statistically significant sample of nearby $(z<0.25)$ clusters. A handful of clusters not contaminated by cooling cores and at $z<0.1$ could be enough to shed light on this issue. This should be kept in mind for the future generation satellites which should have a larger field of view and the same or better resolution and sensitivity than the present X-ray telescopes.

An additional effect to take into account since it could mimic evolution is the presence of the Malmquist bias. With the available archival samples it is very difficult to keep under control and factor out this bias. It would be extremely useful to observe with Chandra or XMM-Newton complete samples of nearby and distant clusters all coming from the same survey with well defined selection criteria in order to be able to control and eventually correct for the bias.

Acknowledgements. We acknowledge stimulating discussions with Stefano Ettori, Ben Maughan, Paolo Tozzi and Anna Wolter. We are grateful to an anonymous referee whose comments contributed to improve this paper. This research made use of data obtained from the Chandra Data Archive, which is part of the Chandra X-Ray Observatory Science Center, operated for the National Aeronautics and Space Administration (NASA) by the Smithsonian Astrophysical Observatory. Partial financial support for this work came from the Italian Space Agency ASI (Agenzia Spaziale Italiana) through grant ASI-INAF $\mathrm{I} / 023 / 05 / 0$.

\section{References}

Allen, S. W. 2003, Ap\&SS, 285, 247

Arnaud, M., \& Evrard, A. E. 1999, MNRAS, 305, 631

Balogh, M. L., Babul, A., \& Patton, D. R. 1999, MNRAS, 307, 463

Borgani, S. 2006, [arXiv: astro-ph/0605575]

Borgani, S., Governato, F., Wadsley, J., et al. 2002, MNRAS, 336, 409

Borgani, S., Murante, G., Springel, V., et al. 2004, MNRAS, 348, 1078

Bower, R. G., Benson, A. J., Lacey, C. G., et al. 2001, MNRAS, 325, 497

Branchesi, M., Gioia, I. M., Fanti, C., Fanti, R. 2007a, A\&A, 472, 727 (Paper I)

Branchesi, M., Gioia, I. M., Fanti, C., Fanti, R., \& Cappelluti, N. 2007b, A\&A, 462, 449

Brighenti, F., \& Mathews, W. G. 2001, ApJ, 553, 103

Bryan, G. L. 2000, ApJ, 544, L1

Bryan, G. L., \& Norman, M. L. 1998, ApJ, 495, 80

Cavaliere, A., \& Fusco-Femiano, R. 1976, A\&A, 49, 137

Ettori, S., Tozzi, P., Borgani, S., \& Rosati, P. 2004, A\&A, 417, 13 (ET04)

Evrard, A. E., \& Henry, J. P. 1991, ApJ, 383, 95

Evrard, A. E., Metzler, C. A., \& Navarro, J. F. 1996, ApJ, 469, 494

Evrard, A. E., Macfarland, T. J., Couchman, H. M. P., et al. 2002, ApJ, 573, 7

Fabian, A. C., Crawford, C. S., Edge, A. C., \& Mushotzky, R. F. 1994, MNRAS, 267, 779

Kaiser, N. 1986, MNRAS, 222, 323

Kaiser, N. 1991, ApJ, 383, 104

Kotov, O., \& Vikhlinin, A. 2005, ApJ, 633, 781

Markevitch, M. 1998, ApJ, 504, 27

Maughan, B. J. 2007, [arXiv:astro-ph/0703504]

Maughan, B. J., Jones, L. R., Ebeling, H., \& Scharf, C. 2006, MNRAS, 365, 509 (MA06)

Maughan, B. J., Jones, C., Forman, W., \& Van Speybroeck, L. 2007, [arXiv:astro-ph/0703156]

Muanwong, O., Kay, S. T., \& Thomas, P. A. 2006, ApJ, 649, 640

Ponman, T. J., Cannon, D. B., \& Navarro, J. F. 1999, Nature, 397, 135

Reiprich, T. H., \& Böhringer, H. 2002, ApJ, 567, 716

Spitzer, L., Jr. 1978, Physical Processes in the Interstellar Medium (New York: Wiley)

Tozzi, P. 2006, [arXiv: astro-ph/0602072]

Tozzi, P., \& Norman, C. 2001, ApJ, 546, 63

Tornatore, L., Borgani, S., Springel, V., et al. 2003, MNRAS, 342, 1025

Vikhlinin, A., VanSpeybroeck, L., Markevitch, M., Forman, W. R., \& Grego, L. 2002, ApJ, 578, L107 (VI02)

Voit, G. M. 2005a, Rev. Mod. Phys. 77, 207

Voit, G. M. 2005b, Adv. Space Res., 36, 701

Voit, G. M., \& Bryan, G. L. 2001, Nature, 414, 425

Voit, G. M., Bryan, G. L., Balogh, M. L., \& Bower, R. G. 2002, ApJ, 576, 601

Wu, X.-P., \& Xue, Y.-J. 2002, ApJ, 569, 112 